\title{
Sufficient conditions for close-to-starlikeness and close-to-convexity of order $\beta$
}

\author{
Yuwei Liu ${ }^{1, a}$, Lifeng Guo ${ }^{2, b}$ \\ ${ }^{1}$ College of Petroleum Engineering, Northeast Petroleum University \\ Daqing 163318, China. \\ ${ }^{2}$ School of Mathematical Science and Technology, Northeast Petroleum University \\ Daqing 163318, China. \\ 1yuweiliuhl@126.com, 2 hitglf@yahoo.com.cn
}

Keywords: Analytic functions; Close-to-star functions; Close-to-convex functions.

Abstract. The object of the present paper is to obtain certain sufficient conditions for close-to-starlikeness and close-to-convexity of order $\beta$.

\section{Introduction}

The function, for which the equation $f(z)=w$ has $p$ roots in $D$ for every complex number $w$, is said to be $p$-valent (or multivalent) function, where $D$ is a domain in the extended complex plane $C$. Let $H$ be the class of analytic functions in $U=\{z \in C:|z|<1\}$, and $A_{p}$ be the subclass of $H$ consisting of functions of the form

$$
f(z)=z^{p}+a_{p+1} z^{p+1}+a_{p+2} z^{p+2}+\cdots, p \in N, z \in U
$$

with $A_{1}=A$.

A function $f(z) \in A_{p}$ is consist as starlike of order $\alpha(0 \leq \alpha<p)$ in $U$ (see [1]), that is, $f(z) \in S_{p}^{*}(\alpha)$, if and only if

$$
\operatorname{Re}\left(\frac{z f^{\prime}(z)}{f(z)}\right)>\alpha, 0 \leq \alpha<p, z \in U
$$

with $S_{1}^{*}(0):=S^{*}$.

A function $f(z) \in A_{p}$ is consist as $p$-valently strongly close-to-star of order $\beta(0<\beta \leq 1)$ in $U$ with respect to $g(z)$, that is, $f(z) \in C S_{p}^{*}(\beta)$, if and only if

$$
\left|\arg \left(\frac{f(z)}{g(z)}\right)\right|<\frac{\beta \pi}{2}, z \in U
$$

for some real $\beta(0<\beta \leq 1)$ and for some starlike function $g(z) \in A_{p}$ (see [2]). For $g(z)=z^{p}$ in condition (3), we have that $f(z) \in A_{p}$ is $p$-valently strongly close-to-star of order $\beta(0<\beta \leq 1)$ in $U$ if

$$
\left|\arg \left(\frac{f(z)}{g(z)}\right)\right|<\frac{\beta \pi}{2}, z \in U
$$

A function $f(z) \in A_{p}$ is consist as $p$-valently strongly close-to-convex of order $\beta(0<\beta \leq 1)$ in $U$ with respect to $g(z)$, that is, $f(z) \in C C_{p}(\beta)$, if and only if

$$
\left|\arg \left(\frac{z f^{\prime}(z)}{g(z)}\right)\right|<\frac{\beta \pi}{2}, z \in U
$$

for some real $\beta(0<\beta \leq 1)$ and for some starlike function $g(z) \in A_{p}$ (see [3]). For $g(z)=z^{p}$ in condition (5), we have that $f(z) \in A_{p}$ is $p$-valently strongly close-to-convex of order $\beta(0<\beta \leq 1)$ in $U$ if 


$$
\left|\arg \left(\frac{z f^{\prime}(z)}{g(z)}\right)\right|<\frac{\beta \pi}{2}, z \in U
$$

In proving our main theorem, we need the following lemma due to Owa, Nunokawa, Saitoh and Fukui.

Lemma 1.1. (see [4])Let Let $p(z)$ be analytic, $p(z) \neq 0$ in $U$ and $p(0)=1$. Suppose that there exists a point $z_{0} \in U$ such that

$$
|\arg p(z)|<\frac{\pi \beta}{2} \text { for }|z|<\left|z_{0}\right|
$$

and

$$
\left|\arg p\left(z_{0}\right)\right|=\frac{\pi \beta}{2}
$$

where $\beta>0$. Then we have

$$
\frac{z_{0} p^{\prime}\left(z_{0}\right)}{p\left(z_{0}\right)}=i k \beta
$$

where

$$
k \geq \frac{1}{2}\left(\gamma+\frac{1}{\gamma}\right) \geq 1 \text { when } \arg p\left(z_{0}\right)=\frac{\pi \beta}{2}
$$

and

$$
k \leq-\frac{1}{2}\left(\gamma+\frac{1}{\gamma}\right) \leq-1 \text { when } \arg p\left(z_{0}\right)=-\frac{\pi \beta}{2}
$$

where

$$
p\left(z_{0}\right)^{1 / \beta}= \pm i \gamma \text { and } \gamma>0 .
$$

\section{Main Result}

Theorem 2.1. If $f(z) \in A_{p}$ satisfies the condition,

$$
\left|1+\frac{z f^{\prime \prime}(z)}{f^{\prime}(z)}-\alpha\right|<\alpha, z \in U
$$

where

$$
\alpha=\frac{p^{2}+\beta^{2}}{2 p}
$$

and $0<\beta \leq 1$, then $f(z) \in C C_{p}(\beta)$.

Proof. Let us put

$$
F(z)=\frac{f^{\prime}(z)}{p z^{p-1}}
$$

By logarithmic differentiation of (15), we have

$$
\frac{z F^{\prime}(z)}{F(z)}=1+\frac{z f^{\prime \prime}(z)}{f^{\prime}(z)}-p
$$

or

$$
\frac{z F^{\prime}(z)}{F(z)}+p=1+\frac{z f^{\prime \prime}(z)}{f^{\prime}(z)}
$$

Suppose there exist a point $z_{0} \in U$ such that

$$
|\arg F(z)|<\frac{\pi}{2} \beta \text { for }|z|<\left|z_{0}\right|
$$

and

$$
\left|\arg F\left(z_{0}\right)\right|=\frac{\pi}{2} \beta,
$$


then from Lemma 1.1, we have

$$
\frac{z_{0} F^{\prime}\left(z_{0}\right)}{F\left(z_{0}\right)}=i \beta k
$$

where

$$
k \geq \frac{1}{2}\left(\gamma+\frac{1}{\gamma}\right) \geq 1 \text { when } \arg p\left(z_{0}\right)=\frac{\pi \beta}{2}
$$

and

$$
k \leq-\frac{1}{2}\left(\gamma+\frac{1}{\gamma}\right) \leq-1 \text { when } \arg p\left(z_{0}\right)=-\frac{\pi \beta}{2}
$$

where

$$
p\left(z_{0}\right)^{1 / \beta}= \pm i \gamma \text { and } \gamma>0 .
$$

At first, let us suppose $p\left(z_{0}\right)^{1 / \beta}=i \gamma$, then we have

$$
\frac{z_{0} F^{\prime}\left(z_{0}\right)}{F\left(z_{0}\right)}+p=i \beta k+p
$$

where

$$
k \geq \frac{1}{2}\left(\gamma+\frac{1}{\gamma}\right) \geq 1
$$

From this, we have

$$
\operatorname{Re}\left(\frac{z_{0} F^{\prime}\left(z_{0}\right)}{F\left(z_{0}\right)}+p\right)^{-1}=\frac{p}{p^{2}+\beta^{2} k^{2}} \leq \frac{p}{p^{2}+\beta^{2}}
$$

Since $|w-\alpha|<\alpha \Leftrightarrow \operatorname{Re}(1 / w)>\frac{1}{2 \alpha}$, this contradicts the assumption of this theorem.

For the case $p\left(z_{0}\right)^{1 / \beta}=-i \gamma$, applying the same method as the above, we have the condition (26). Therefore we complete the proof.

Theorem 2.2. If $f(z) \in A_{p}$ satisfies the condition,

where

$$
\left|\frac{z f^{\prime}(z)}{f(z)}-\alpha\right|<\alpha, z \in U
$$

$$
\alpha=\frac{p^{2}+\beta^{2}}{2 p}
$$

and $0<\beta \leq 1$, then $f(z) \in S_{p}^{*}(\beta)$.

Proof. Let us put

$$
F(z)=\frac{f(z)}{z^{p}} .
$$

By logarithmic differentiation of (15), we have

$$
\frac{z F^{\prime}(z)}{F(z)}=\frac{z f^{\prime}(z)}{f(z)}-p
$$

or

$$
\frac{z F^{\prime}(z)}{F(z)}+p=\frac{z f^{\prime}(z)}{f(z)}
$$

Suppose there exist a point $z_{0} \in U$ such that

$$
|\arg F(z)|<\frac{\pi}{2} \beta \text { for }|z|<\left|z_{0}\right|
$$

and

$$
\left|\arg F\left(z_{0}\right)\right|=\frac{\pi}{2} \beta,
$$


then from Lemma 1.1, we have

$$
\frac{z_{0} F^{\prime}\left(z_{0}\right)}{F\left(z_{0}\right)}=i \beta k
$$

where

$$
k \geq \frac{1}{2}\left(\gamma+\frac{1}{\gamma}\right) \geq 1 \text { when } \arg p\left(z_{0}\right)=\frac{\pi \beta}{2}
$$

and

$$
k \leq-\frac{1}{2}\left(\gamma+\frac{1}{\gamma}\right) \leq-1 \text { when } \arg p\left(z_{0}\right)=-\frac{\pi \beta}{2}
$$

where

$$
p\left(z_{0}\right)^{1 / \beta}= \pm i \gamma \text { and } \gamma>0 .
$$

At first, let us suppose $p\left(z_{0}\right)^{1 / \beta}=i \gamma$, then we have

where

$$
\frac{z_{0} F^{\prime}\left(z_{0}\right)}{F\left(z_{0}\right)}+p=i \beta k+p
$$

$$
k \geq \frac{1}{2}\left(\gamma+\frac{1}{\gamma}\right) \geq 1
$$

From this, we have

$$
\operatorname{Re}\left(\frac{z_{0} F^{\prime}\left(z_{0}\right)}{F\left(z_{0}\right)}+p\right)^{-1}=\frac{p}{p^{2}+\beta^{2} k^{2}} \leq \frac{p}{p^{2}+\beta^{2}}
$$

Since $|w-\alpha|<\alpha \Leftrightarrow \operatorname{Re}(1 / w)>\frac{1}{2 \alpha}$, this contradicts the assumption of this theorem.

For the case $p\left(z_{0}\right)^{1 / \beta}=-i \gamma$, applying the same method as the above, we have the condition (26). Therefore we complete the proof.

\section{References}

[1] M. S. Robertson, On the theory of univalent functions, Ann. of Math., 37 (1936), 374-408.

[2] Y. O. Park and S. Y. Lee, On a class of strongly close-to-star functions, Bull. Korean Math. Soc., 37(4) (2000), 755-764.

[3] H. Shiraishi and S. Owa, Some sufficient problems for Strongly Close-to-Convex of order $\mu$, General Mathematics, 17 (4) (2009), 157-169.

[4] S. Owa, M. Nunokawa, H. Saitoh and S. Fukui, Starlikeness and close-to-convexity of certain analytic functions, Far East J. Math. Sci. 2(2) (1994), 143-148. 